\title{
OTIMIZAÇÃO DA DESIDRATAÇÃO OSMÓTICA DA HASTE CENTRAL DE ABACAXI PÉROLA UTILIZANDO A METODOLOGIA DE SURPERFÍCIE DE RESPOSTA
}

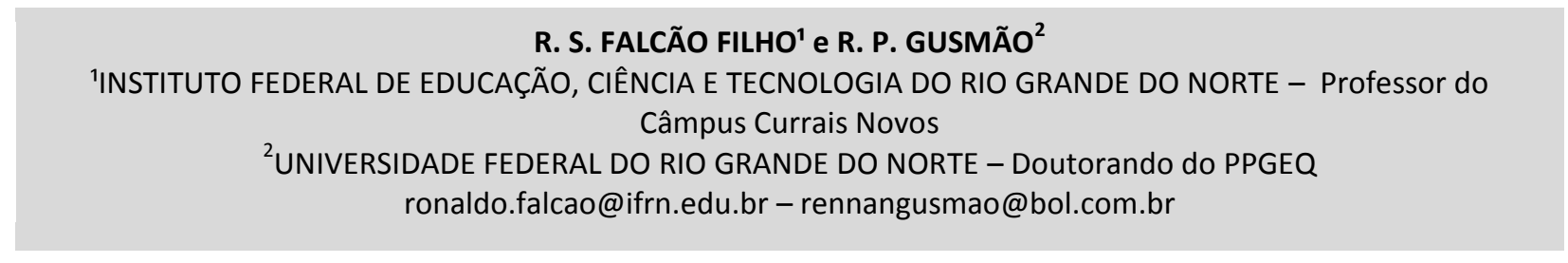

Artigo submetido em agosto/2012 e aceito em setembro/2012

\section{RESUMO}

O presente artigo teve como objetivo otimizar o processo de desidratação osmótica de fatias de hastes centrais de abacaxis pérola a partir das variáveis de processo: temperatura, concentração da solução osmótica e proporção fruta-solução osmótica. A desidratação osmótica (D.O.) de fatias de talo de abacaxi em soluções de sacarose foi realizada em estufa com circulação de ar e estudada mediante planejamento experimental fatorial $2^{3}$ com 4 repetições no ponto central, totalizando 12 experimentos. As três variáveis independentes para o processo foram: temperatura $(T)$ e concentração da solução osmótica (C) e proporção fruta-solução osmótica (F:SO).
Realizou-se o ajuste de modelo matemático aos pontos experimentais por meio de regressão linear e a avaliação do deste através da análise de variância (ANOVA), do teste de Fischer (F) para a regressão e para a falta de ajuste e do coeficiente de correlação $\left(R^{2}\right)$. O modelo foi considerado preditivo, razoavelmente ajustado e os seus resíduos, em relação aos pontos experimentais, se distribuíram de forma aproximadamente normal. A partir dessas confirmações o modelo foi considerando válido e a otimização do processo, na região estudada, deu-se em: $\mathrm{T}=50$ oC; C $=40 \mathrm{~g} .100 \mathrm{~g}^{-1}$ e F:SO = 3:20.

PALAVRAS-CHAVE: ananas comosus (L.) Meril, desidratação osmótica, planejamento experimental, metodologia de superfície de resposta.

\section{OPTIMIZATION OF OSMOTIC DEHYDRATION OF PINEAPPLE PEARL CENTER ROD USING RESPONSE SURFACE METHODOLOGY}

\section{ABSTRACT}

This article aimed to optimize the process of osmotic dehydration of stem central of sliced pineapple pearl from process variables: temperature, osmotic solution concentration and proportion fruit-osmotic solution. Osmotic dehydration (O.D) of sliced pineapple stem in sucrose solutions was performed in an oven with air circulation and studied by experimental design 23 factorial with 4 replications at the center point, totaling 12 experiments. The three independent variables for the process were: temperature $(T)$ and osmotic solution concentration (C) and proportion fruit-osmotic solution ( $\mathrm{F}: \mathrm{SO})$.
We carried out the adjustment of the mathematical model to the experimental data using linear regression and evaluation of this by analysis of variance (ANOVA) and test Fischer (F) for the regression fit and lack of correlation coefficient (R2). The model was considered predictive, reasonably adjusted and their residues with respect to the experimental points, if so approximately normal distributed. From these confirmations, the model was considered valid and process optimization the region studied occurred in: $\mathrm{T}=50^{\circ} \mathrm{C}, \mathrm{C}=40 \mathrm{~g} .100 \mathrm{~g}^{-1}$ and $\mathrm{F}: \mathrm{S}=3: 20$

KEY-WORDS: ananas comosus (L.) Meril, osmotic dehydration, experimental planning, response surface methodology. 


\section{OTIMIZAÇÃO DA DESIDRATAÇÃO OSMÓTICA DA HASTE CENTRAL DE ABACAXI PÉROLA UTILIZANDO A METODOLOGIA DE SURPERFÍCIE DE RESPOSTA}

\section{INTRODUÇÃO}

O abacaxizeiro (Ananas comosus (L.) Meril) é uma planta perene, monocotiledônea, pertencente à família Bromeliaceae, que contém aproximadamente 1900 espécies de plantas herbáceas. O fruto é múltiplo (sincarpo) e resulta do desenvolvimento da inflorescência, contendo de 100 a 200 flores individuais que evoluem individualmente, estando inseridos sobre uma haste central, que é uma extensão do pedúnculo, em disposição espiralada e intimamente soldadas entre si. Em seu ápice há um tufo de folhas, ou coroa. A forma do fruto é cilíndrica ou cônica, e sua massa varia entre 1 a 3 kg (MEDINA,1987; BRASIL, 1999; FAO, 2009).

O abacaxi por longo tempo tem sido a fruta, não pertencente à família citrus, mais popular nos países tropicais e subtropicais, principalmente pelo seu sabor e aroma atrativos, além de conter grande diversidade de vitaminas e sais minerais, com destaque para a vitamina $C$ e para o potássio (VALENTE, 2007).

A produção nacional de abacaxi no ano de 2008 foi de aproximadamente 1,7 bilhão de unidades. Deste montante, o Nordeste e a Paraíba produziram cerca de 788 e 345 milhões de unidades, respectivamente, tornando-se a região e o estado com a maior produção (SIDRA, 2009).

Infelizmente existe grande desperdício dessa produção em nosso país e por isso, métodos de conservação devem ser adotados para que isto seja evitado. O Ministério da Agricultura considerou em relatório publicado 1993 que o Brasil perde mais de 1 bilhão de dólares por ano com o desperdício de frutas e hortaliças e que as perdas do abacaxi chegam a $23 \%$ da produção (BRASIL, 1993)

Um método de conservação bastante interessante para o abacaxi é a desidratação osmótica, um processo brando de conservação, que vem sendo estudado durante décadas e que pode ser aplicado como etapa preliminar ou adicional a outros processamentos, por ser capaz de remover parte da água presente nos alimentos sem severas agressões de sua natureza estrutural, nutricional e funcional. A desidratação osmótica, combinada a métodos complementares de secagem, apresenta-se como um ótimo método de conservação para frutas e, além disto, agrega um bom valor ao produto que pode ser produzido facilmente por pequenos produtores rurais (SOUSA, 2008; FALCÃO FILHO, 2011).

Para otimizar o processo de desidratação osmótica pode-se utilizar um planejamento experimental. De acordo com Rodrigues e lemma (2005), o planejamento experimental, baseado na estatística, é sem dúvidas, uma ferramenta poderosa na otimização e desenvolvimento de processos e formulações ou, simplesmente, para avaliar impactos que certos fatores têm sobre determinadas respostas.

A necessidade de se encontrar medidas para reduzir o desperdício de insumos e partes comestíveis de matérias-primas alimentares, por meio da utilização dessas partes e da racionalização do uso dos insumos, justifica a realização deste estudo. Sendo assim, o objetivo do trabalho foi otimizar o processo de desidratação osmótica de fatias de hastes centrais de abacaxis pérola a partir das variáveis de processo: temperatura, concentração da solução osmótica e proporção fruta-solução osmótica. 


\section{MATERIAL E MÉTODOS}

O presente trabalho foi desenvolvido no laboratório de processamento de produtos de origem vegetal do IFRN Câmpus Currais Novos. Foram utilizados como matéria-prima abacaxis da variedade pérola, da safra de 2010, provenientes da zona rural da região metropolitana de João Pessoa-PB (latitude: $7^{\circ} 5^{\prime} 0^{\prime \prime} \mathrm{S}$ e longitude: $34^{\circ} 50^{\prime} 0^{\prime \prime} \mathrm{W}$ ) e adquiridos no comércio de Currais Novos-RN (latitude: 6 $6^{\circ} 15^{\prime} 39^{\prime \prime}$ S e longitude: $36^{\circ} 30^{\prime} 54^{\prime \prime} \mathrm{W}$ ) .

O processo de produção de passa da haste central do abacaxi pérola foi complementado por uma secagem solar indireta em secador desenvolvido no IFRN Câmpus Currais Novos para que o teor de água ficasse em conformidade com a legislação, que estabelece teor de umidade máximo de $25 \%$ para frutas desidratadas (BRASIL, 1978). O processo de secagem e a eficiência do secador foram estudados monitorando-se as temperaturas do coletor solar, da câmara de secagem e do ambiente, tempo de secagem, além da velocidade do ar de secagem que foi.

A desidratação osmótica (D.O.) de fatias de talo de abacaxi em soluções de sacarose foi estudada mediante planejamento experimental fatorial $2^{3}$ com três variáveis independentes: temperatura $(T)$, concentração da solução osmótica $(C)$ e proporção fruta-solução osmótica (F:SO), avaliadas em dois níveis $(-1$ e +1$)$ e com quatro repetições nos pontos centrais (0), totalizando 12 experimentos (BARROS NETO; SCARMINIO; BRUNS, 2010; RODRIGUES; IEMMA, 2005; CALADO; MONTGOMERY, 2003). Todos os experimentos foram realizados em triplicata e tiveram duração de $4 \mathrm{~h}$, sem agitação.

Determinou-se em testes preliminares que o modelo que melhor se ajusta às varáveis estudadas neste trabalho é o linear, por este motivo, não se utilizou um delineamento composto central rotacional (DCCR), delineamento tal, composto por pontos axiais, além dos fatoriais e central que gerariam um modelo quadrático.

As respostas foram os parâmetros: perda de água (PA), ganho de sólidos (GS) e a relação ganho de sólidos-perda de água (GS:PA) que foi a variável de otimização utilizada neste estudo.

As faixas estudadas das variáveis temperatura e concentração do xarope foram definidas segundo Almeida et al. (2005). A faixa proporção fruta-solução osmótica foi definida baseando-se em Valente (2007) o qual afirma que uma boa proporção fruta é a de 1:10, pois esta garante que a concentração do xarope seja constante durante todo processo de desidratação. Assim, a proporção 1:10 foi adotada como ponto central para a variável proporção fruta-solução osmótica e a metade desta, ou seja, 1:20 o ponto fatorial inferior $(-1)$ e 1,5 vezes o ponto central o limite superior $(+1)$, ou seja, 3:20.

Os experimentos foram realizados segundo os níveis descritos na Tabela 1 e no planejamento estabelecido na Tabela 2.

Tabela 1. Variáveis independentes e níveis do planejamento

\begin{tabular}{lccc}
\hline Variáveis & \multicolumn{3}{c}{ Níveis } \\
\cline { 2 - 4 } independentes & -1 & 0 & +1 \\
\hline $\mathrm{T}(\stackrel{\mathrm{o}}{ } \mathrm{C})$ & 30 & 40 & 50 \\
$\mathrm{C}\left(\mathrm{g} .100 \mathrm{~g}^{-1}\right)$ & 40 & 50 & 60 \\
$\mathrm{~F}: \mathrm{SO}$ & $1: 20$ & $1: 10$ & $3: 20$ \\
\hline
\end{tabular}

Nota: $-1,0$ e +1 representam a codificação dos níveis. 
Tabela 2. Planejamento experimental $2^{3}$ com 4 repetições no ponto central

\begin{tabular}{llll}
\hline Ensaio & $\mathrm{T}(\underline{\mathrm{o}} \mathrm{C})$ & $\mathrm{C}(\mathrm{g} .100 \mathrm{~g}-1)$ & $\mathrm{F}: \mathrm{SO}$ \\
\hline 1 & $30(-1)$ & $40(-1)$ & $1: 20(-1)$ \\
2 & $30(-1)$ & $40(-1)$ & $3: 20(+1)$ \\
3 & $30(-1)$ & $60(+1)$ & $3: 20(+1)$ \\
4 & $30(-1)$ & $60(+1)$ & $1: 20(-1)$ \\
5 & $50(+1)$ & $40(-1)$ & $1: 20(-1)$ \\
6 & $50(+1)$ & $40(-1)$ & $3: 20(+1)$ \\
7 & $50(+1)$ & $60(+1)$ & $3: 20(+1)$ \\
8 & $50(+1)$ & $60(+1)$ & $1: 20(-1)$ \\
9 & $40(0)$ & $50(0)$ & $1: 10(0)$ \\
10 & $40(0)$ & $50(0)$ & $1: 10(0)$ \\
11 & $40(0)$ & $50(0)$ & $1: 10(0)$ \\
12 & $40(0)$ & $50(0)$ & $1: 10(0)$ \\
\hline
\end{tabular}

Os experimentos de desidratação foram realizados em estufa com circulação de ar modelo 420-D da NOVA ÉTICA, onde as fatias de haste de abacaxi foram desidratadas em soluções de sacarose nas concentrações descritas no planejamento e contidas em recipientes de vidro. Os recipientes permaneceram fechados durante a desidratação osmótica para que o sistema fruta-solução não perdesse água para o ar de circulação da estufa, dessa maneira, conservando-se a massa de água total do sistema.

O procedimento de avaliação da desidratação osmótica foi realizado de acordo com Dionello et al. (2007). Depois de retiradas das soluções osmóticas, as amostras foram enxaguadas em água destilada por cerca de 5 segundos com a finalidade de retirar o excesso de soluto retido na superfície do produto, sendo posteriormente drenadas em papel toalha para retirada do excesso de água superficial.

Logo após a desidratação osmótica, as amostras foram analisadas segundo o seu conteúdo de umidade (IAL, 2008).

Os cálculos de perda de água, ganho de sólidos e relação ganho de sólidos-perda de água, no processo de desidratação osmótica, foram obtidos por meio das equações indicadas por Almeida et al. (2005).

$$
\begin{gathered}
\mathrm{PA}=\frac{\left(\mathrm{M}_{0}-\mathrm{M}_{\mathrm{t}}\right) \cdot 100}{\mathrm{~W}_{0}} \\
\mathrm{GS}=\frac{\left(\mathrm{MS}_{\mathrm{t}}-\mathrm{MS}_{0}\right) \cdot 100}{\mathrm{~W}_{0}}
\end{gathered}
$$$$
\text { Eq. } 1
$$

Em que,

$M_{0}$ - Massa inicial de água da amostra (g)

$\mathrm{M}_{\mathrm{t}}$ - Massa de água da amostra no tempo $\mathrm{t}(\mathrm{g})$

$\mathrm{MS}_{0}$ - Massa inicial de sólidos na amostra (g)

$\mathrm{MS}_{\mathrm{t}}$ - Massa de sólidos na amostra no tempo $\mathrm{t}(\mathrm{g})$

$\mathrm{W}_{0}$ - Massa da amostra (g) 
Um modelo matemático linear foi ajustado aos pontos experimentais utilizando-se o programa Statistica versão 7.0. A partir da análise de variância (ANOVA) do modelo linear testouse o poder de predição do modelo por meio do teste de Fischer (F) para a regressão. Já a falta de ajuste do modelo foi testada através do teste $\mathrm{F}$ para a falta de ajuste e pelo coeficiente de correlação $\left(R^{2}\right)$ (BARROS NETO; SCARMINIO; BRUNS, 2010). Os testes de ajuste e predição do modelo foram realizados ao nível de significância de $5 \%$.

A normalidade da distribuição dos resíduos, do modelo em relação aos pontos experimentais, foi testada graficamente observando-se a proximidade e a distribuição dos resíduos em torno da reta de valores normais (MOORE, 2011).

A escolha do ponto ou da faixa ideal de otimização do processo foi feita utilizando-se a metodologia da superfície de resposta, através observação da superfície de resposta e de sua curva de nível, ambas geradas pelo programa estatístico e que são as representações gráficas da equação ajustada pela regressão linear, cujo modelo geral é mostrado pela Equação 3.

$$
y=\beta_{0}+\beta_{1} \cdot x_{1}+\beta_{2} \cdot x_{2}+\beta_{3} \cdot x_{3}+\beta_{1,2} \cdot x_{1} \cdot x_{2}+\beta_{1,3} \cdot x_{1} \cdot x_{3}+\beta_{2,3} \cdot x_{2} \cdot x_{3}+\varepsilon \quad \text { Eq. } 3
$$

Em que,

y - Variável dependente, no caso: GS:PA

$\beta_{i}$ - Coeficientes do modelo

$\mathrm{x}_{\mathrm{i}}$ - Variáveis independentes, no caso: T, C e F:SO

\section{RESULTADOS E DISCUSSÃO}

A maximização da perda de água e perda de peso com o mínimo de absorção de solutos é o principal objetivo da otimização da desidratação osmótica.

Valente (2007), afirma que a maximização da perda de água não é suficiente para alcançar as melhores condições de processamento, uma vez que isso resultaria em produtos com alta incorporação de solutos. Em contrapartida, uma minimização do ganho de sólidos resultaria num produto com baixa perda de água.

Então o parâmetro que foi utilizado na otimização da desidratação osmótica foi a razão GS:PA, pois este é facilmente interpretado da seguinte forma: quando o ganho de sólidos tende ao seu valor mínimo e a perda de água ao seu máximo a relação GS:PA tende a zero.

É possível observar na Tabela 3 que a temperatura foi o único efeito significativo para a razão GS:PA, isso é mostrado pelo p-valor menor do que 0,05. Esse fato é extremamente importante para a otimização do processo, pois mostra que se pode usar a concentração mínima e a maior relação fruta:solução reduzindo-se os custos de utilização de sacarose na desidratação osmótica, e preservando ao máximo as características de fruta in natura do produto.

Observando-se a Tabela 3, também se pode identificar que o efeito da temperatura é negativo, demonstrando que a razão GS:PA decresce com o aumento da temperatura, dessa forma, a GS:PA tende a um valor mínimo com o aumento de temperatura. 
Tabela 3. Efeitos da razão perda de água-ganho de sólidos (GS:PA) avaliados pelo erro-padrão.

\begin{tabular}{|c|c|c|c|c|c|c|}
\hline \multirow[b]{2}{*}{ Fatores } & \multirow[b]{2}{*}{ Efeitos } & \multirow[b]{2}{*}{$\begin{array}{l}\text { Erro- } \\
\text { padrão }\end{array}$} & \multirow[b]{2}{*}{$\mathrm{t}_{(3)}$} & \multirow[b]{2}{*}{ p-valor } & \multicolumn{2}{|c|}{$\begin{array}{c}\text { Estimativa por } \\
\text { intervalo }\end{array}$} \\
\hline & & & & & $\begin{array}{c}\text { Limite de } \\
\text { confiança } \\
\text { inferior }\end{array}$ & $\begin{array}{l}\text { Limite de } \\
\text { confiança } \\
\text { superior }\end{array}$ \\
\hline Média & 0,3393 & 0,0054 & 62,4477 & 0,0000 & 0,3220 & 0,3566 \\
\hline $\mathrm{T}(\stackrel{\mathrm{o}}{\mathrm{C}})$ & $-0,1063$ & 0,0133 & $-7,9866$ & 0,0041 & $-0,1486$ & $-0,0639$ \\
\hline C (\%) & 0,0251 (ns) & 0,0133 & 1,8895 & 0,1552 & $-0,0172$ & 0,0675 \\
\hline $\mathrm{F}: \mathrm{SO}$ & $-0,0176$ (ns) & 0,0133 & $-1,3188$ & 0,2789 & $-0,0599$ & 0,0248 \\
\hline $\mathrm{T} \times \mathrm{C}$ & 0,0018 (ns) & 0,0133 & 0,1327 & 0,9029 & $-0,0406$ & 0,0441 \\
\hline $\mathrm{T} \times \mathrm{F}: \mathrm{SO}$ & 0,0067 (ns) & 0,0133 & 0,5023 & 0,6500 & $-0,0357$ & 0,0490 \\
\hline CxF:SO & $-0,0159$ (ns) & 0,0133 & $-1,1961$ & 0,3176 & $-0,0583$ & 0,0264 \\
\hline
\end{tabular}

Nota: (ns) valor não significativo ao nível de $5 \%$ de probabilidade.

Depois do descarte de todos os fatores e interação de fatores não significativos procedeuse uma análise de variância e calculou-se o coeficiente de determinação do modelo estatístico linear para a razão GS:PA. Os resultados estão expressos na Tabela 4.

A ANOVA mostra que o modelo ajustado aos pontos experimentais é preditivo. A predição do modelo foi constatada pelo fato do $F_{\text {calc }}$ da regressão linear ser aproximadamente dez vezes maior do que o $F_{t a b}$, segundo Rodrigues e lemma (2005); Barros Neto, Scarminio e Bruns (2010) o valor de $F_{\text {calc }}$ deve ser quatro ou cinco vezes maior do que o $F_{\text {tab }}$ para que o modelo seja considerado preditivo.

Analisando-se a Tabela 4 também é possível concluir que o modelo tem uma falta de ajuste não significativa e um $R^{2}$ de aproximadamente 0,82 , estes resultados confirmam que 0 modelo é razoavelmente bem ajustado os valores experimentais.

Tabela 4. Análise de variância (ANOVA) do modelo ajustado para GS:PA.

\begin{tabular}{ccccc}
\hline Fontes de variação & SQ & GL & QM & $F_{\text {calc }}$ \\
\hline Regressão linear & 0,0226 & 1 & 0,0226 & 49,1239 \\
Resíduos & 0,0047 & 10 & 0,0005 & \\
Falta de ajuste & 0,0036 & 7 & 0,0005 & 1,4571 \\
Erro puro & 0,0011 & 3 & 0,0004 & \\
SQ & 0,0272 & 11 & & \\
\hline
\end{tabular}

$F_{1 ; 10-0,05}=4,96 ; F_{7 ; 3-0,05}=8,89$ e $R^{2}=0,8294$

Como o modelo utilizado neste trabalho tem como base a estatística paramétrica, um pressuposto importante que deve ser verificado é a normalidade dos resíduos da regressão, felizmente a ANOVA é uma técnica bastante robusta e pequenos desvios de normalidade não a comprometem (MOORE, 2011). Essa normalidade pode ser verificada por meio de testes de normalidade ou graficamente, como pode ser visto na Figura 1. A figura mostra que os resíduos se distribuem razoavelmente próximos da normalidade que é representada pela reta no gráfico, dessa forma, não comprometendo o pressuposto de normalidade da ANOVA. 


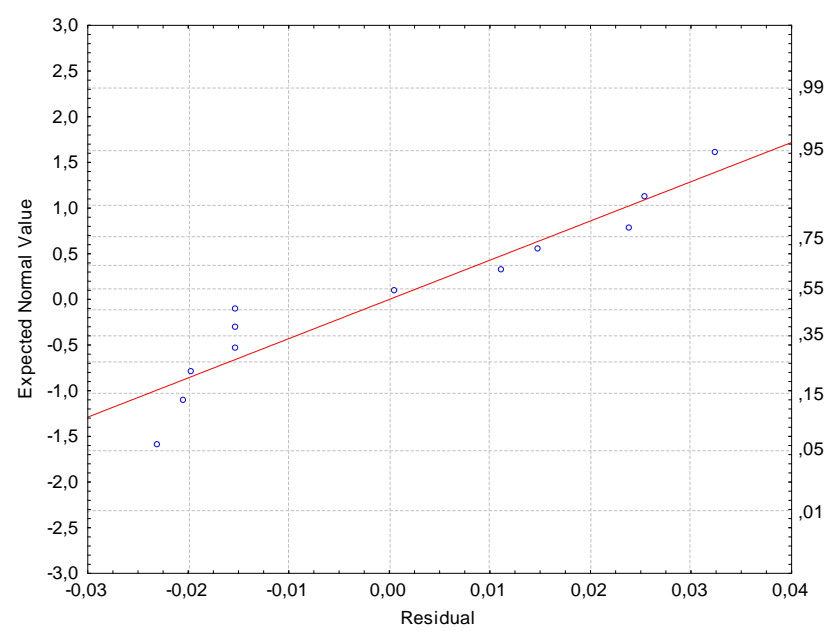

Figura 1. Distribuição dos resíduos dos valores do planejamento.

Como o modelo foi considerado preditivo, bem ajustado e obediente ao pressuposto de normalidade dos resíduos, a Equação 4 representa o modelo linear para a relação GS/PA em função da temperatura do processo, o termo independente e o coeficiente da temperatura estão acompanhados de seus respectivos erros-padrão, os valores estão apresentados na forma codificada.

$$
G S / P A=(0,3393 \pm 0,0054)+(-0,0531 \pm 0,067) . T \quad \text { Eq. } 4
$$

Analisando-se a Figura 2 (a) fica evidente que a desidratação osmótica é ótima na região de temperatura (+1) 50 C, quando a razão GS:PA é mínima, não importando a concentração da solução, a relação $\mathrm{F}: \mathrm{SO}$, nem qualquer interação de efeitos, dessa forma, o processo ficou otimizado com a temperatura no nível mais elevado, concentração da solução osmótica no nível mais baixo e relação fruta-solução também no nível mais baixo, já que concentração e relação fruta-solução não foram variáveis significantes no processos, para a região estudada.
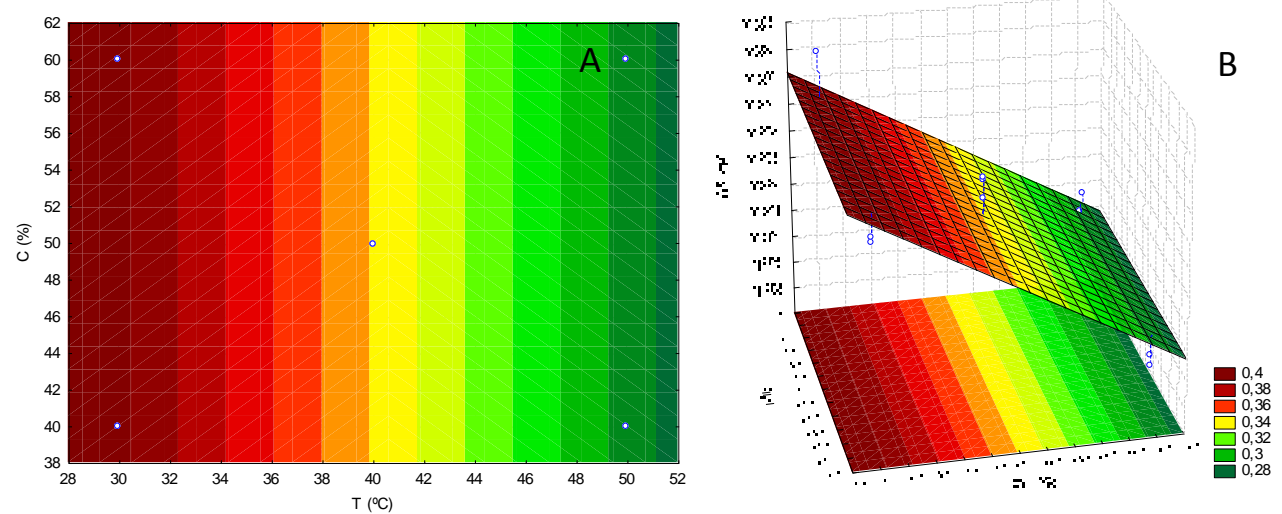

Figura 2. (a) curva de nível e (b) superfície de resposta da razão GS:PA em função da temperatura.

Como a única variável que se mostrou significativa para o processo na região estudada foi a temperatura a otimização da desidratação dar-se-á de acordo com os valores expostos na Tabela 5. Para economizar sacarose no processo osmótico adotam-se a menor concentração de solução de desidratação e a maior proporção fruta-solucão osmótica. 
Tabela 5. Valores otimizados para a desidratação osmótica.

\begin{tabular}{lcr}
\hline Variável de processo & Valor codificado & Valor real \\
\hline $\mathrm{T}(\stackrel{\mathrm{o}}{ } \mathrm{C})$ & +1 & 50 \\
$\mathrm{C}\left(\mathrm{g} .100 \mathrm{~g}^{-1}\right)$ & -1 & 40 \\
$\mathrm{~F}: \mathrm{SO}$ & -1 & $3: 20$ \\
\hline
\end{tabular}

\section{CONCLUSÕES}

O modelo estatístico escolhido apresentou-se preditivo e razoavelmente ajustado para a variável de otimização ganho de sólidos-perda de água (GS:PA), além disso, os resíduos se distribuíram de forma aproximadamente normal, a partir destas confirmações pode-se afirmar que o modelo, de fato, representa a GS:PA em função da temperatura de processo.

A otimização da desidratação osmótica deu-se com a minoração da razão GS:PA chegando-se a combinação com maior perda de água, menor ganho de sólidos e economia de açúcar. Para essa combinação otimizada as variáveis de processo tiveram os valores ótimos em: 50 C de temperatura, $40 \mathrm{~g} \cdot 100^{-1} \mathrm{~g}$ de concentração da solução de desidratação e uma proporção de fruta-solução de 3:20.

\section{REFERÊNCIAS}

1. ALMEIDA, Francisco de A. C. et al .Otimização do processo de secagem osmótica na obtenção de produtos secos da manga Tommy Atkins. Revista Brasileira Engenharia Agrícola e Ambiental, Campina Grande, dez. 2005, v. 9, n. 4, p.576-584.

2. BARROS NETO, Benício de; SCARMINIO, leda Spacino; BRUNS, Roy Edward. Como fazer experimentos: Pesquisa e desenvolvimento na ciência e na indústria. 4. ed. Porto Alegre: Bookman, 2010. p.414.

3. BRASIL. Getúlio Augusto Pinto da Cunha. Embrapa (Org.). O Abacaxizeiro: cultivo, agroindústria e economia. Brasília: Embrapa, 1999. p.480.

4. BRASIL. Ministério da Agricultura do Abastecimento e da Reforma Agrária. Perdas na agropecuária brasileira: relatório preliminar da comissão técnica para redução das perdas na agropecuária. Brasília, 1993.

5. BRASIL. Cnnpa. Resolução no 12: Aprova regulamento técnico de identidade e qualidade de frutas secas. 24 jul. 1978. Disponível em: http://www.anvisa.gov.br/legis/resol/12_78_frutas_secas.htm. Acesso em 02 jan. 2011.

6. CALADO, Verônica; MONTGOMERY, Douglas. Planejamento de experimentos usando Statistica: Pesquisa e desenvolvimento na ciência e na indústria. Rio de Janeiro: E-papers Serviços Editoriais, 2003. p.260.

7. DIONELLO, Rafael Gomes et al . Desidratação por imersão-impregnação de abacaxi em soluções de sacarose e em xarope de açúcar invertido. Ciência e Tecnologia de Alimentos, dez. 2007, vol.27, no.4, p.701-709.

8. FAO, Food and Agriculture Organization of the United Nations. Disponível em: < www.fao.org>. Acesso em 10 de nov. 2009. 
9. FALCÃO FILHO, Ronaldo Dos Santos. Produção de passas de talo de abacaxi pérola por processo osmo-solar. 2011. 72 f. Dissertação (Mestrado) - Ufpb, João Pessoa, 2011.

10. INSTITUTO ADOLFO LUTZ. Métodos físico-químicos para análise de alimentos. São Paulo: Instituto Adolfo Lutz, 2008. p.1020.

11. MEDINA, J. C. Capítulo I: cultura. Série frutas tropicais: abacaxi. 2 ed. Campinas: ITAL, 1987. p.1-132.

12. MOORE, David. A estatística básica e sua prática. 5. ed. Rio de Janeiro: Ltc, 2011. 668p.

13. RODRIGUES, Maria Isabel; IEMMA, Antônio Francisco. Planejamento de experimentos e otimização de processos: uma estratégia sequencial de planejamentos. Ed. Campinas Campinas-SP, 2005.

14. SIDRA, sistema IBGE de recuperação automática. Disponível em: www.sidra.ibge.br. Acesso em 10 nov. 2009.

15. SOUSA, Severina de. Obtenção de figos secos por desidratação osmótica e secagem convectiva. 2008. [s.n.]. Tese (Doutorado) - Unicamp, Campinas, 2008.

16. SOUZA NETO, Manoel Alves de et al. Desidratação osmótica de manga seguida de secagem convencional: avaliação das variáveis de processo. Ciência e Agrotecnologia., Out 2005, vol.29, no.5, p.1021-1028.

17. VALENTE, Pedro Paulo Sanches da Silva. Desidratação osmótica e secagem de abacaxi (Ananás Comosus (L.) Merril), variedade pérola. 2007. [s.n.]. Dissertação (Mestrado) Unicamp, Campinas, 2007. 